\title{
Spectrophotometric Analysis of Preservatives and Coloring Agents in Orange Soft Drink and Orange Juice Samples
}

\author{
Saiful Islam Bhuyian, Tofael Ahmed, M Kamrul Hasan, Mohammad Shoeb* and Nilufar Nahar \\ Department of Chemistry, Dhaka University, Dhaka-1000, Bangladesh
}

(Received: 15 April 2018 ; Accepted: 10 July 2018)

\begin{abstract}
A rapid and simple UV-Vis spectrophotometric method was used for the determination of the level of sodium benzoate and yellow 6 in 14 different batches of orange soft drink samples and 7 different batches of orange juice samples commercially available in local markets of Bangladesh. The standard samples of sodium benzoate and yellow 6 in water were used to make calibration curves and the correlation coefficient $\left(\mathrm{r}^{2}\right)$ of the calibration curves were found to be 0.9993 and 0.9953 for sodium benzoate and yellow 6, respectively. The wavelength of absorption maxima was $225 \mathrm{~nm}$ for Sodium benzoate and $429 \mathrm{~nm}$ for yellow 6. All 14 analyzed soft drinks samples contained yellow 6 and sodium benzoate in the range of 2.90$162.51 \mathrm{mg} / \mathrm{L}$ and 94.06-387.66 mg/L, respectively. The highest amount of sodium benzoate and yellow 6 were found in the sample AMR (387.66 mg/L) and BRC (162.51 mg/L), respectively. Among the 7 orange juice samples yellow 6 were found in the range of $28.03-102.66 \mathrm{mg} / \mathrm{L}$ and the highest amount was in the sample OJ1 $(102.66 \mathrm{mg} / \mathrm{L})$. The reproducibility and repeatability of the method was very satisfactory with low value of RSD in a range of 0.05 to $4.17 \%$ and 0.05 to $4.07 \%$, respectively.
\end{abstract}

Keywords: Preservatives, Yellow 6, Sodium benzoate, Coloring agents.

\section{Introduction}

Carbonated orange drinks are known as orange soft drinks and the liquid extracts of orange are known as orange juice, made by squeezing the fresh orange. To make more attractive and tasty, artificial color and synthetic sweetener are added in the commercial orange soft drinks and juices. Some of the additives are beneficial and some are harmful for health. ${ }^{1}$ Orange soft drinks often contain high levels of sodium benzoate and it imparts a slight metallic taste to the soft drinks. Other additive that is generally found in beverage is yellow 6 (Disodium 6-hydroxy-5-(4sulfophenyl)azo-2-naphthalenesulfonate), known as sunset yellow. ${ }^{1}$ Usually, only yellow 6 is found in orange juice which is used as color additives.

People often suffer from asthmatic attacks, hives or other allergic reactions when consume drinks/juices containing with excessive sodium benzoate. It is also responsible for having blood cancer when it gets associated with ascorbic acid. $^{2}$ On the other hand, there are some assuming fact that aspirin intolerance people suffer allergic reaction with various symptoms such as nettle rash (urticaria), diarrhea, gastric upset, migraines, swelling of the skin (angioedema) and vomiting that are caused by yellow $6 .{ }^{3}$ Yellow 6 and other artificial colorings were found to affect children behavior especially hyperactivity. ${ }^{4}$ In the present study, we are now reporting sodium benzoate and yellow 6 in locally marketed orange soft drinks and orange juices.

\section{Experimental}

\section{Collection of samples}

Fourteen commercial batches of orange soft drinks and seven orange juice samples were collected from different markets of Dhaka city during June 2015 and March 2016.

\section{Chemical and solvents}

All chemical and reagents used were of analytical grade. Sulfuric acid (98\%, w/w, BDH, U.K.), phenol (Merck, Mumbai, India), de-ionized water were used during project work.

\section{Standard Chemicals}

Analytical standard of sodium benzoate, yellow 6 and glucose for reference were collected from Drug Administration Bangladesh and stored at $0{ }^{\circ} \mathrm{C}$ in a refrigerator.

\section{Preparation of standard solutions}

The primary standard solution of sodium benzoate (100 $\mathrm{mg} / \mathrm{L}$ ) was prepared in HPLC grade water. Working standard solutions $(75.0,50.0,20.0,10.0,5.0,4.0$ and 2.0 $\mathrm{mg} / \mathrm{L}$ ) of sodium benzoate were prepared from there. Similarly, the working standard solutions $(20.0,10.0,5.0$, 4.0, 2.0 and $1.0 \mathrm{mg} / \mathrm{L}$ ) of yellow 6 were also prepared from primary standard solution $(100 \mathrm{mg} / \mathrm{L})$ of yellow 6 . Absorbances of these solutions were measured by a double beam UV-Visible spectrophotometer at 225 and $429 \mathrm{~nm}$ and calibration curves were made for sodium benzoate and yellow 6 , respectively. The primary standard solution of glucose $(100 \mathrm{mg} / \mathrm{L})$ was prepared in HPLC grade water. The working standard solutions $(80.0,60.0,50.0,30.0$ and $15.0 \mathrm{mg} / \mathrm{L}$ ) were prepared from primary standard solution.

\section{Test for carbohydrate}

Glucose solution $(500 \mu \mathrm{l})$ was taken in the test tube followed by addition of $80 \%$ aqueous phenol $(50 \mu \mathrm{L})$ and then it was vortexed. After that, conc. $\mathrm{H}_{2} \mathrm{SO}_{4}(3 \mathrm{~mL})$ was added to the mixture. A UV- Visible spectrophotometer was used to measure the absorbance $(478 \mathrm{~nm})$ of each of the solution. By plotting absorbance versus glucose concentration a calibration curve was made. 


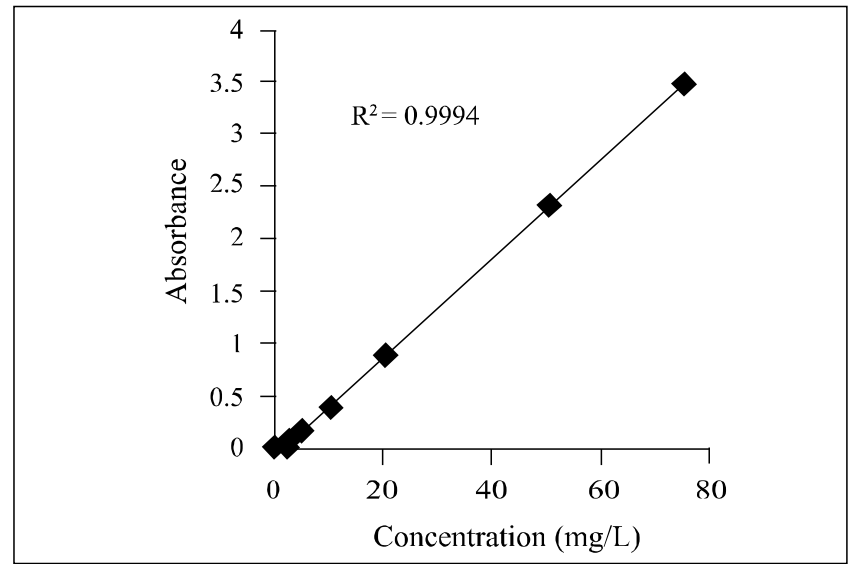

(a)

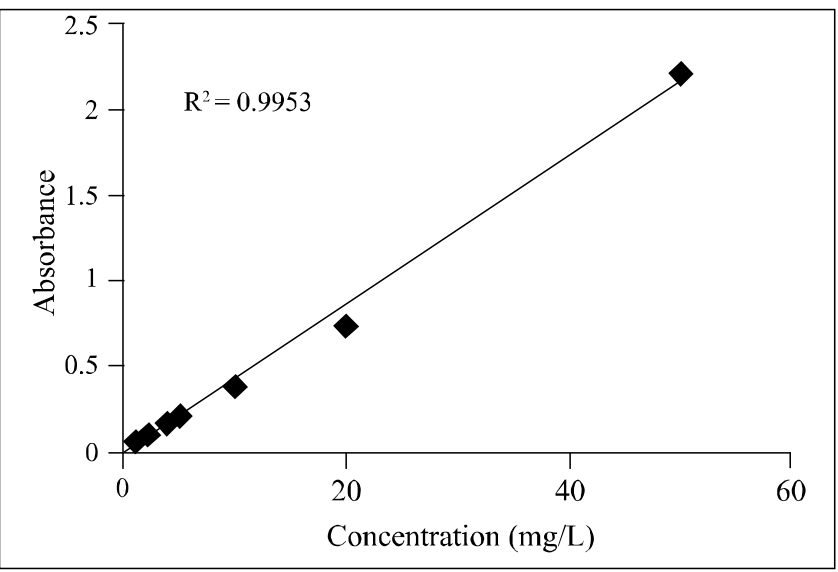

(b)

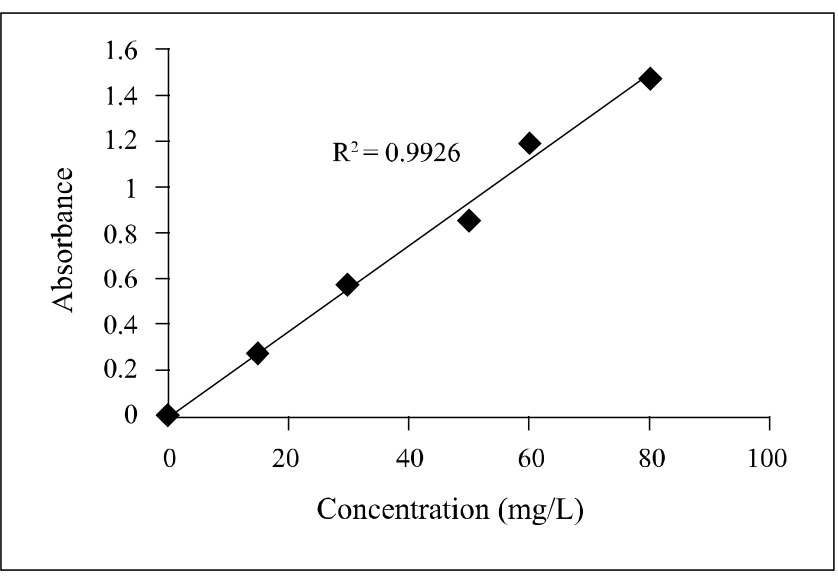

(c)

Fig. 1. Calibration curves for standard (a) sodium benzoate (b) yellow 6 and (c) glucose solution

\section{Preparation of sample solutions}

The orange soft drink samples $(100 \mathrm{~mL})$ were filtered with HPLC grade filter paper and degassed in a degasser for 30 minutes and the volume of the sample after degassing was recorded. The samples of orange soft drinks and orange juice were then diluted by a factor of ten. The solutions were homogeneously mixed with the help of a vortex machine and shaking was carried out for two minutes. The absorbance of the prepared sample solution from direct orange soft drink and orange juice were then measured.

Table1. Amount of sample (orange soft drinks) taken for degassing and estimation of the amount of dissolved gas.

\begin{tabular}{|c|c|c|c|}
\hline $\begin{array}{c}\text { Sample } \\
\text { code }\end{array}$ & $\begin{array}{c}\text { Initial volume } \\
(\mathbf{m L})\end{array}$ & $\begin{array}{c}\text { Final volume } \\
(\mathbf{m L})\end{array}$ & $\begin{array}{c}\text { Volume of } \\
\text { dissolved gas } \\
(\mathbf{m L})\end{array}$ \\
\hline AMR & 100 & 93.50 & 6.50 \\
BMR & 100 & 93.20 & 6.80 \\
CMR & 100 & 94.00 & 6.00 \\
AF & 100 & 92.50 & 7.50 \\
BF & 100 & 93.00 & 7.00 \\
CF & 100 & 94.00 & 6.00 \\
AUR & 100 & 92.00 & 8.00 \\
BUR & 100 & 92.40 & 7.60 \\
CUR & 100 & 92.80 & 7.20 \\
ARC & 100 & 93.00 & 7.00 \\
BRC & 100 & 92.80 & 7.20 \\
CRC & 100 & 93.00 & 7.00 \\
AT & 100 & 91.80 & 8.20 \\
BT & 100 & 91.00 & 9.00 \\
\hline
\end{tabular}

\section{Determination of total carbohydrate}

Total carbohydrate in orange soft drink and juice solutions were determined by performing modified Molisch's method and using the standard calibration curve made for glucose. Approximately $2-3 \mathrm{~mL}$ of the sample was taken in a test tube and $50 \mu \mathrm{L} 80 \%$ aqueous phenol was added to it. Then the mixer was mixed well by a vortex for $30 \mathrm{sec}$ after that 3 $\mathrm{mL}$ of concentrated sulfuric acid $(98 \%)$ was added to the mixer and mixed thoroughly by a vortex for $1 \mathrm{~min}$. After 30 minutes reddish-brown color was developed and the solution was then taken in a $25 \mathrm{~mL}$ volumetric flask. The volume was adjusted with concentrated sulfuric acid (98\%) and absorbance of the solution was determined.

\section{Determination of $\mathrm{pH}$ value}

About $3 \mathrm{~mL}$ orange soft drink and juice sample from each batch was taken in a beaker and $30 \mathrm{~mL}$ water was added. Then $\mathrm{pH}$ of each sample was determined by using Microprocessor $\mathrm{pH}$ meter (Model: HANNA pH 211).

\section{Results and Discussion}

Analysis of samples

Analysis of sodium benzoate and yellow 6 were carried out in all samples by UV-Visible Spectrophotometric method. Sodium benzoate and yellow 6 were found in all batches of orange soft drinks whereas only yellow 6 were found in orange juice samples. Different batches of orange soft drinks contained sodium benzoate in a range of 94-387 $\mathrm{mg} / \mathrm{L}(0.094 \%$ to $0.387 \%)$ and yellow 6 contained within the range of $0.0029 \%$ to $0.162 \%$. The allowable limit of sodium benzoate and yellow 6 are $0.1 \%$ and $0.3 \%$ by weight, respectively. ${ }^{[5,6]}$ The range of yellow 6 in different batches of orange juice samples were $0.028 \%$ to $0.102 \%$. Whereas the allowable limit of yellow 6 is $0.3 \%$ by weight. ${ }^{7}$ 
Table2. The amount of sodium benzoate and yellow 6 in different batch of samples.

\begin{tabular}{|c|c|c|c|c|c|}
\hline $\begin{array}{c}\text { Sample } \\
\text { name }\end{array}$ & Code & $\begin{array}{c}\text { Amount of } \\
\text { sodium } \\
\text { benzoate } \\
(\mathrm{mg} / \mathrm{L})\end{array}$ & $\begin{array}{l}\text { RSD } \\
(\%)\end{array}$ & $\begin{array}{l}\text { Amount of } \\
\text { yellow } 6 \\
(\mathrm{mg} / \mathrm{L})\end{array}$ & $\begin{array}{c}\text { RSD } \\
(\%)\end{array}$ \\
\hline \multirow{14}{*}{$\begin{array}{l}\text { Orange } \\
\text { soft } \\
\text { drinks. }\end{array}$} & AMR & $387.66 \pm 0.213$ & 0.05 & $103.79 \pm 0.54$ & 0.52 \\
\hline & BMR & $355.60 \pm 28.68$ & 4.07 & $94.20 \pm 0.62$ & 0.66 \\
\hline & CMR & $304.68 \pm 0.213$ & 0.07 & $68.55 \pm 0.54$ & 0.79 \\
\hline & $\mathrm{AF}$ & $94.04 \pm 0.43$ & 0.45 & $3.10 \pm 0.61$ & 4.17 \\
\hline & $\mathrm{BF}$ & $104.54 \pm 0.32$ & 0.31 & $29.75 \pm 0.25$ & 0.82 \\
\hline & $\mathrm{CF}$ & $163.47 \pm 0.32$ & 0.20 & $102.91 \pm 0.24$ & 0.24 \\
\hline & AUR & $275.32 \pm 0.21$ & 0.08 & $55.94 \pm 0.62$ & 1.11 \\
\hline & BUR & $373.76 \pm 0.032$ & 0.09 & $79.01 \pm 0.25$ & 0.31 \\
\hline & CUR & $180.28 \pm 0.54$ & 0.38 & $37.61 \pm 0.71$ & 1.87 \\
\hline & ARC & $374.47 \pm 0.21$ & 0.06 & $78.60 \pm 0.99$ & 1.26 \\
\hline & BRC & $306.38 \pm 0.21$ & 0.07 & $162.51 \pm 1.07$ & 0.66 \\
\hline & $\mathrm{CRC}$ & $280.71 \pm 0.44$ & 0.16 & $157.01 \pm 0.52$ & 0.32 \\
\hline & AT & $224.89 \pm 0.26$ & 0.26 & $16.45 \pm 0.65$ & 3.95 \\
\hline & BT & $153.26 \pm 0.25$ & 0.16 & $12.35 \pm 0.62$ & 4.05 \\
\hline \multirow{7}{*}{$\begin{array}{l}\text { Orange } \\
\text { juice }\end{array}$} & OJ1 & - & - & $102.66 \pm 0.25$ & 0.24 \\
\hline & $\mathrm{OJ} 2$ & - & - & $79.75 \pm 0.24$ & 0.31 \\
\hline & $\mathrm{OJ} 3$ & - & - & $67.19 \pm 0.26$ & 0.37 \\
\hline & OJ4 & - & - & $36.89 \pm 0.74$ & 2.00 \\
\hline & OJ5 & - & - & $28.03 \pm 0.25$ & 0.88 \\
\hline & OJ6 & - & - & $100.68 \pm 0.27$ & 0.25 \\
\hline & OJ7 & - & - & $88.38 \pm 2.50$ & 3.67 \\
\hline
\end{tabular}

Total carbohydrate

The range of carbohydrate found in orange soft drinks were 10.88-14.36 $\mathrm{g} / \mathrm{L}$, whereas the commercial brand samples of orange soft drinks contained in the range of 10-13 g/L. On the other hand, the range of carbohydrate that was found in orange juice samples were 11.72-13.75 g/L. Higher value of carbohydrates in orange soft drink and juice causes obesity to the consumers.

\section{$p H$}

The orange soft drinks and orange juices are acidic and $\mathrm{pH}$ is around 3.50. $\mathrm{pH}$ of commercial brand orange soft drinks and juice samples were found to be in the range of 2.252.45 and 4.01-4.81, respectively.

Table 3. The value of $\mathrm{pH}$ and the total amount of carbohydrate in each sample.

\begin{tabular}{|c|c|c|c|c|c|}
\hline \multicolumn{3}{|c|}{ Orange soft drinks } & \multicolumn{3}{c|}{ Orange juice } \\
\hline Code & $\mathrm{pH}$ & $\begin{array}{c}\text { Total } \\
\text { carbohydrate } \\
(\mathrm{mg} / \mathrm{L})\end{array}$ & Code & $\mathrm{pH}$ & $\begin{array}{c}\text { Total } \\
\text { carbohydrate } \\
(\mathrm{mg} / \mathrm{L})\end{array}$ \\
\hline AMR & 2.26 & 11.72 & OJ1 & 3.12 & 11.72 \\
\hline BMR & 2.25 & 12.31 & OJ2 & 3.24 & 12.31 \\
\hline CMR & 2.30 & 12.73 & OJ3 & 3.20 & 12.73 \\
\hline AF & 2.41 & 13.15 & OJ4 & 3.15 & 13.15 \\
\hline BF & 2.42 & 13.52 & OJ5 & 2.96 & 13.52 \\
\hline CF & 2.45 & 13.75 & OJ6 & 3.47 & 13.75 \\
\hline AUR & 2.30 & 10.88 & OJ7 & 3.42 & 11.88 \\
\hline BUR & 2.30 & 11.47 & - & - & - \\
\hline CUR & 2.30 & 11.89 & - & - & - \\
\hline ARC & 2.38 & 13.99 & - & - & - \\
\hline BRC & 2.39 & 14.36 & - & - & - \\
\hline CRC & 2.38 & 14.35 & - & - & - \\
\hline AT & 2.35 & 13.48 & - & - & - \\
\hline BT & 2.40 & 13.49 & - & - & - \\
\hline
\end{tabular}

\section{Conclusions}

Fourteen orange soft drinks and seven orange juice samples were analyzed for quantitative determination of sodium benzoate and yellow 6 using UV-Visible spectrometric method. Sodium benzoate and yellow 6 contained all the orange soft drink samples. Yellow 6 contained only seven orange juice samples.

The range of sodium benzoate and yellow 6 in orange soft drink samples were $0.094 \%$ to $0.387 \%$ and $0.0029 \%$ to $0.162 \%$, respectively whereas the range of yellow 6 in orange juice samples were $0.028 \%$ to $0.102 \%$. The allowable limit for sodium benzoate and yellow 6 is $0.1 \%$ and $0.3 \%$ by weight, respectively. Here it can be concluded that yellow 6 found in both type of samples are in satisfactory level but sodium benzoate in orange soft drinks crossed the allowable limit.

\section{Acknowledgement}

Authors are grateful to the International Science Programme (ISP), Uppsala University, Sweden and Higher Education Quality Enhancement Project (HEQEP) for financial supports.

\section{References}

1. Sultana A., M. S. Haque, M. Shoeb, M. S. Islam, M. I. R. Mamun, N. Nahar, 2012. Presence of yellow 6, an artificial colour additive inorange juice, Journal of Bangladesh Chemical Society, 25(1), 80-86

2. Vivekreddy M., G. Aruna, S. Angalaparameswari, B. Haseena Banu, P. Jayachandrareddy, 2015. Estimated daily intake and exposure of sodium benzoate and potassium sorbate through food products in school children of tirupati, india, International Journal of Pharmacy and Pharmaceutical Sciences ISSN-0975-1491, 7, Issue 7

3. Ibero M., J. L. Eseverri, C. Barroso, J. Botey, 1982. Allergol Immunopathol(Madr) 10(4): 263-8

4. Schultz-Ehrenburg U., O. Gilde, 1987. 62 Suppl1:88-95

5. Bronaugh R. L., R. F. Stewart and E. R. Congdon, 1982. Journal of Toxicology and Applied Pharmacology, 62, 481

6. Coverly J., L. Peters, E. Whittle and D. A. Basketter, 1998. Contact Dermatitis, 38, 90

7. Health Canada, 2015, "List of Permitted Food Additives". 
